\title{
Factores asociados a Chlamydia trachomatis en mujeres con vaginitis atendidas en atención primaria en salud
}

\author{
Factors associated with Chlamydia trachomatis in women with vaginitis attending in \\ primary health care
}

Angélica Melo ${ }^{1,2,7}$, Ximena Ossa1,3,6, Luis Bustos ${ }^{1,3,6}$, Giselle Fetis ${ }^{1,4,6}$, Lorena Lazo ${ }^{1,4}$ y Flery Fonseca-Salamanca ${ }^{1,5,7}$

\author{
${ }^{1}$ Facultad de Medicina. Universidad de La Frontera. Temuco, Chile. \\ 2Departamento Anatomía Patológica. \\ ${ }^{3}$ Departamento Salud Pública. \\ ${ }^{4}$ Departamento Obstetricia y Ginecología. \\ ${ }^{5}$ Departamento Ciencias Preclínicas. \\ ${ }^{6}$ Centro de Excelencia Capacitación, Investigación y Gestión para la Salud Basada en Evidencias (CIGES). \\ ${ }^{7}$ Laboratorio de Inmunoparasitología Molecular, Centro de Excelencia en Medicina Traslacional (CEMT-BIOREN).
}

Los autores no tienen conflictos de interés que declarar.

Este estudio fue financiado por la Universidad de La Frontera a través de los Proyectos DI15-0047 y DI17-0123.

Recibido: 21 de septiembre de 2020 / Aceptado: 20 de mayo de 2021

\section{Resumen}

Introducción: Chlamydia trachomatis es reconocida como el agente causal de una de las infecciones de transmisión sexual (ITS) más común y curable. En mujeres, puede ser gatillante de enfermedad inflamatoria pélvica, un factor de riesgo para infertilidad o adquisición de otras ITS. Objetivo: Determinar C. trachomatis en mujeres con diagnóstico clínico de vaginitis asociando factores sociodemográficos, sexuales, clínicos y microbiota vaginal. Pacientes y Métodos: Participaron 121 mujeres voluntarias $>18$ años, con diagnóstico clínico de vaginitis y descarga vaginal anormal, atendidas en servicios de atención primaria en salud (APS) en Temuco-Chile. Se tomó muestra de flujo vaginal, determinándose C. trachomatis por una reacción de polimerasa en cadena convencional y microbiota vaginal por tinción de Gram. Se colectaron datos clínicos, sociodemográficos y sexuales mediante encuesta. Resultados: Rango de edad 18-61 años. La frecuencia de C. trachomatis fue $14,9 \%$. Se asoció C. trachomatis a mayor número de parejas sexuales en los últimos 5 años $(\mathrm{p}=0,001)$, relaciones sexuales oral más anal $(\mathrm{p}=0,055)$ y juguetes sexuales $(\mathrm{p}=0,027)$. Asociación no significativa hubo con vaginosis bacteriana $31,2 \%(\mathrm{p}=0,091)$ e inicio de la actividad sexual $<18$ años $20,6 \%$ $(p=0,07)$. Conclusiones: Sería beneficioso incorporar en la semiología ginecológica de las mujeres atendidas en servicios de APS, los factores encontrados como favorecedores de infección por C. trachomatis, pues ello podría ayudar al manejo de esta ITS.

Palabras clave: Chlamydia trachomatis; mujeres adultas; vaginitis; parejas sexuales; coinfección.

\section{Abstract}

Background: Chlamydia trachomatis is recognized as the causative agent of one of the most common and curable sexually transmitted infections (STIs). In women, it can be a trigger for pelvic inflammatory disease, a risk factor for infertility or acquisition of other STIs. Aim: To determine CT in women with vaginitis and associate it with sociodemographic, sexual, clinical, and vaginal microbiota factors. Methods: 121 female volunteers $>18$ years of age, with a clinical diagnosis of vaginitis and abnormal vaginal discharge, attending in primary health care services (PHC) in Temuco-Chile were recluted. A vaginal discharge sample was taken, determining $C$. trachomatis by polymerase chain reaction and vaginal microbiota by Gram stain. Clinical, sociodemographic and sexual data were collected by survey. Results: Age range was 18-61 years. The frequency of $C$. trachomatis was $14.9 \%$. C. trachomatis was associated with a higher number of sexual partners in the last 5 years $(\mathrm{p}=0.001)$, oral plus anal sex $(p=0.055)$ and sex toys $(p=0.027)$; there was a non-significant association with bacterial vaginosis $31.2 \%(\mathrm{p}=0.091)$ and initiation of sexual activity $<18$ years $20.6 \%(p=0.07)$. Conclusions: It would be beneficial to incorporate in the gynecological semiology of the women attended in PHC services, the factors found to favor C. trachomatis infection, as this could help the management of this STI.

Keywords: Chlamydia trachomatis; adult women; vaginitis; sexual partners; coinfection.

\footnotetext{
Correspondencia a:

Angélica Melo Angermeyer (ORCID: 0000-0002-3576-1745)
}

angelica.melo@ufrontera.cl 


\section{Introducción}

C

hlamydia trachomatis es una bacteria de multiplicación obligada intracelular, reconocida como el agente causal de una de las infecciones de transmisión sexual (ITS) más común y curable ${ }^{1}$. Puede ser un factor de riesgo para la adquisición de otras ITS y llegar a afectar gravemente la salud reproductiva de las mujeres ${ }^{2}$. Se ha reportado que la infección por $C$. trachomatis es un factor de riesgo para infertilidad y una causa importante de enfermedad inflamatoria pélvica (EIP). Hoenderboom y cols., en un estudio de seguimiento de ocho años reportaron un riesgo considerablemente más alto de EIP e infertilidad por factor tubárico en mujeres con infección por C. trachomatis ${ }^{3}$ y Price y cols., observaron un riesgo de EIP (sintomática o asintomática) de $17,1 \%$ post una infección de C. trachomatis sin tratamiento 4 .

En relación a factores de riesgo, existe evidencia de asociación de infección por C. trachomatis en mujeres bajo 25 años de edad y bajo nivel socioeconómico ${ }^{5}$, también con un gran número de parejas $(>5)$ en el último año ${ }^{6}$ y con sexo extragenital ${ }^{7}$; además, factores sociodemográficos como un nivel educacional universitario han sido asociados con un riesgo de positividad a C. trachomatis ${ }^{8}$.

En Chile, en los últimos años se han publicado algunos estudios, en la Región Metropolitana y en La Araucanía, en grupos de mujeres que muestran similares diferencias en las frecuencias de C. trachomatis, excepto el estudio de Ovalle y cols., realizado en mujeres embarazadas entre 14-43 años, el que reporta una presencia de $C$. trachomatis de $5,9 \%{ }^{9-12}$.

Mostrar evidencia con respecto a este tema, desde diferentes enfoques y poblaciones se hace imprescindible para crear estrategias y programas para el manejo de esta infección. Una revisión sistemática a estudios relacionados a ITS (clamidiasis, gonorrea, tricomoniasis y sífilis), considera que la prevalencia e incidencia global permanece alta y los autores instan a recolección de datos para mejorar la estrategia de la Organización Mundial de la Salud (OMS) 2016-2021 ${ }^{13}$.

Como en la Región de la Araucanía los estudios publicados se han realizado en población asintomática ${ }^{9,10}$, se planteó como objetivo determinar presencia de $C$. trachomatis en mujeres usuarias del servicio público de salud, con diagnóstico clínico de vaginitis, que tenían diferentes grados de descarga vaginal y asociarla a factores sociodemográficos, sexuales y estado de la microbiota vaginal.

\section{Pacientes y Método}

\section{Diseño}

Estudio de corte transversal, realizado durante 20182019.

\section{Lugares de recolección}

Centros de salud pública de atención primaria en Temuco, Chile.

\section{Participantes}

Ciento veintiuna mujeres voluntarias mayores de 18 años, con descarga vaginal anormal diagnosticada clínicamente. Los criterios de exclusión fueron: discapacidad cognitiva, tratamiento antimicrobiano en los últimos 30 días o uso de fármacos inmunosupresores, menstruación al momento de la toma de la muestra. Posteriormente se aplicó una encuesta estandarizada (identificada con un código) con la cual se recolectaron datos sociodemográficos, hábitos sexuales y datos clínicos (características del flujo, genitales internos).

\section{Variables}

Datos sociodemográficos: edad, lugar de residencia y nivel educacional. Hábitos sexuales: edad de inicio de actividad sexual, parejas sexuales en los últimos cinco años, parejas actuales (al momento del estudio), relaciones sexuales vaginales, orales y anales, uso de juguetes sexuales, preservativo (como uso preventivo para ITS), anticonceptivo (hormonal, preservativo, otros) y frecuencia de relaciones sexuales (último seis meses). Datos clínicos: cantidad de flujo vaginal, genitales internos (eritema vaginal y cervical), microbiota vaginal y coinfecciones (fueron considerados todos los casos que presentaron: microbiota intermedia, Candida spp, Trichomonas vaginalis y vaginosis bacteriana o una combinación entre ellas, de manera independiente de si tuvieran o no C. trachomatis).

\section{Muestras de flujo vaginal}

Se obtuvieron mediante tórula de algodón estéril de la zona vaginal "fondo de saco" durante un examen de rutina y trasladadas a temperatura ambiente al laboratorio dentro de las $24 \mathrm{~h}$ siguientes, junto al consentimiento informado y la encuesta.

\section{Tinción de Gram}

Se realizó extendido del flujo vaginal sobre un portaobjeto, se observó por microscopia con tinción de Gram aplicando los criterios de Nugent que clasifican la microbiota en normal, intermedia y vaginosis bacteriana. La observación se realizó en 20 campos a objetivo 1000X. Se cuantificaron los morfotipos bacterianos, bacilos grampositivos (Lactobacillus spp.), cocobacilos Gram variable/Gram negativos ( Gardnerella vaginalis/Prevotella spp.) y bacilos Gram variable curvos (Mobiluncus spp.); dependiendo de la cantidad por campos leídos se asignó un puntaje, correspondiendo de 0-3 microbiota normal con prevalencia de Lactobacillus spp, 4-6 intermedia con microbiota mixta y $7-10$ vaginosis bacteriana con predo- 
minio de cocobacilos y escasos o ausente Lactobacillus $\mathrm{spp}^{14}$. Se registró la visualización de blastoconidios y/o pseudohifas.

\section{Extracción del ADN}

Se utilizó el kit E.Z.N.A. ${ }^{\circledR}$ Tissue DNA (Omega BioTek USA), siguiendo las instrucciones del fabricante. Control interno: para descartar muestras con inhibidores de amplificación e integridad del ADN obtenido, se utilizó la reacción de polimerasa en cadena (RPC) simple para el gen beta globina con partidores $\mathrm{PCO} 45$ '-caacttcatcca cgttcacc-3' y GH20 5'-gaagagccaaggacaggtac-3' que amplifican un fragmento de $268 \mathrm{pb}$. Control positivo: ADN genómico.

\section{Detección de C. trachomatis}

Se realizó utilizando partidores KL1 5'-tccggagcgagttacgaaga-3' KL2 5' -aatcaatgcccgggattggt3' (15) que amplifican un fragmento de $241 \mathrm{pb}$ del plásmido críptico de C. trachomatis, mediante RPC simple (RPCCT) con un programa de 35 ciclos y temperatura de hibridación $55^{\circ} \mathrm{C}$. El control positivo fue: muestras clínicas $C$. trachomatis positivas $(\mathrm{CT}(+))$ detectadas por RPC en tiempo real (Abbott RealTime CT/NG assay).

\section{Detección de T. vaginalis}

Se utilizó RPC simple convencional con partidores específicos cuyas secuencias han sido previamente publicadas ${ }^{16}$. Controles positivos: Se usó muestras clínicas positivas detectadas en examen al fresco para T. vaginalis.

\section{Interpretación de resultados}

La observación de una banda de 241pares de bases (pb) para C. trachomatis y de 300pb para T. vaginalis en geles de agarosa teñidos con gel red se consideró positivo.

\section{Aspectos éticos}

Las participantes firmaron un consentimiento informado escrito el cual fue leído, explicado, aclaradas las dudas y entregado una copia. El protocolo de investigación fue autorizado por el Comité Ético Científico de la Universidad de La Frontera (resolución mediante acta 045-2017) y el Comité de Ética Científica del Servicio de Salud Araucanía Sur (resolución mediante carta fechada 2017 noviembre 28). Todas las participantes recibieron tratamiento de acuerdo al protocolo de rutina según cada diagnóstico clínico. Una vez obtenidos los resultados fueron comunicados a las matronas tratantes para su conocimiento y toma de decisiones clínicas.

\section{Análisis estadístico}

Se realizó análisis descriptivo utilizando frecuencias y porcentajes para caracterizar a las participantes y los resultados de laboratorio. Para asociar la presencia de
C. trachomatis y características de las participantes se utilizó prueba exacta de Fisher. El nivel de significancia fue de $5 \%$.

\section{Resultados}

Participaron 121 mujeres con flujo vaginal anormal de distintos centros de salud pública de la comuna de Temuco. El rango de edad estuvo entre 18-61 años con una mediana de 30 años (rango intercuartílico: 24 y 40). La distribución según grupos etarios fue: $28,3 \%, 37,5 \%$ y $34,2 \%$ para $18-24$ años, $25-35$ años y $\geq 36$ años, respectivamente.

El $87,3 \%$ tenía residencia urbana y $66,2 \%$ educación básica-secundaria.

La frecuencia global estimada de C. trachomatis para este estudio fue de 14,9\% (18/121), IC 95\% (9 y 22\%).

En la Tabla 1 se muestran las características sociodemográficas según la presencia de C. trachomatis. Destaca con C. trachomatis $(+)$ el grupo etario $<36$ años $(18,9 \% 15 / 79)$ versus $\geq 36$ años $(7,3 \% 3 / 41)$ no siendo significativa esta diferencia.

En la Tabla 2 se describen algunos hábitos sexuales de las participantes y presencia de C. trachomatis. Se evidencia un porcentaje mayor de $C$. trachomatis en mujeres con más de dos parejas sexuales en los últimos cinco años, tener relaciones sexuales orales más anales y usar juguetes sexuales, siendo estas diferencias significativas.

En la variable inicio de actividad sexual, el grupo antes de los 18 años tuvo un mayor porcentaje de C. trachomatis; sin embargo, esta diferencia no alcanzó significancia estadística con respecto al grupo de mayor edad.

Tabla 1. Características sociodemográficas del grupo de mujeres con vaginitis según la presencia de Chlamydia trachomatis

\begin{tabular}{|c|c|c|c|c|}
\hline \multirow{3}{*}{ Variables } & \multirow{3}{*}{ n } & \multicolumn{2}{|c|}{ Chlamydia trachomatis } & \multirow{3}{*}{$\mathbf{p}$} \\
\hline & & $(-)$ & $(+)$ & \\
\hline & & n (\%) & n (\%) & \\
\hline \multicolumn{5}{|l|}{ Edad (años) } \\
\hline $18-24$ & 34 & $27(79,4)$ & $7(20,6)$ & \\
\hline $25-35$ & 45 & $37(82,2)$ & $8(17,8)$ & \\
\hline$\geq 36$ & 41 & $38(92,7)$ & $3(4,3)$ & 0,21 \\
\hline \multicolumn{5}{|l|}{ Residencia } \\
\hline Rural & 15 & $12(80,0)$ & $3(20,0)$ & \\
\hline Urbana & 103 & $88(85,4)$ & $15(14,6)$ & 0,699 \\
\hline \multicolumn{5}{|l|}{ Nivel educacional } \\
\hline Básica-Secundaria & 80 & $70(87,5)$ & $10(12,5)$ & \\
\hline Técnica-Universitaria & 41 & $33(80,5)$ & $8(19,5)$ & 0,610 \\
\hline
\end{tabular}


Tabla 2. Hábitos sexuales del grupo de mujeres con vaginitis según la presencia de Chlamydia trachomatis

\begin{tabular}{|c|c|c|c|c|}
\hline \multirow{3}{*}{ Variables* } & \multicolumn{4}{|c|}{ Chlamydia trachomatis } \\
\hline & $\mathbf{n}$ & $(-)$ & $(+)$ & p \\
\hline & & n (\%) & n (\%) & \\
\hline \multicolumn{5}{|c|}{ Edad de inicio de actividad sexual (años) } \\
\hline$<18$ & 68 & $54(79,4)$ & $14(20,6)$ & \\
\hline$\geq 18$ & 52 & $48(92,3)$ & $4 \quad(7,7)$ & 0,07 \\
\hline
\end{tabular}

Parejas sexuales

\begin{tabular}{|c|c|c|c|}
\hline 1 & 78 & $73(93,6)$ & $5 \quad(6,4)$ \\
\hline$\geq 2$ & 42 & $29(69,8)$ & $13(30,2)$ \\
\hline
\end{tabular}

Pareja actual

$\begin{array}{rrrrr}\text { Sí } & 110 & 95(86,4) & 15(13,6) & \\ \text { No } & 9 & 6(66,6) & 3(33,3) & 0,136\end{array}$

Relaciones sexuales ${ }^{\#}$

Oral

$\begin{array}{lrrrr}\text { Sí } & 39 & 33(84,6) & 6(15,4) & \\ \text { No } & 54 & 50(92,6) & 4(7,4) & 0,311\end{array}$

Anal

Sí

No

$44(100,0) \quad 0$

Oral + anal

$\begin{array}{lrrrr}\text { Sí } & 26 & 19(73,1) & 7(18,2) & \\ \text { No } & 93 & 83(89,2) & 10(10,8) & 0,055\end{array}$

Juguetes

Sí $95(55,6) \quad 4(44,4)$

$\begin{array}{lllll}\text { No } & 112 & 98(87,5) & 14(12,5) & 0,027\end{array}$

Preservativo preventivo

$\begin{array}{lllll}\text { Sí } & 96 & 84(87,5) & 12(12,5) & \\ \text { No } & 25 & 19(76,0) & 6(24,0) & 0,204\end{array}$

Métodos anticonceptivos

$\begin{array}{lrrrr}\text { Sí } & 72 & 59(81,9) & 13(18,1) & \\ \text { No } & 49 & 44(89,8) & 5(10,2) & 0,302\end{array}$

Frecuencia de relaciones sexuales ${ }^{\&}$

$\begin{array}{lrrrr}\text { No ha tenido } & 3 & 3(100,0) & 0 & \\ <1 \text { vez/semana } & 46 & 37(80,4) & 9(19,6) & \\ \geq 1 \text { vez/semana } & 71 & 62(87,3) & 9(12,7) & 0,653\end{array}$

*Variables: Edad inicio actividad sexual (1 valor perdido), Parejas sexuales (1 valor perdido), Parejas actuales (2 valores perdidos), Relaciones sexuales, orales más anales (2 valores perdidos, uno de ellos Chlamydia trachomatis positivo), Frecuencia relaciones sexuales (1 valor perdido).

\#Últimos 5 años. \&últimos 6 meses.
En la Tabla 3 se muestran algunas características clínicas y coinfecciones (microbiota intermedia, vaginosis bacteriana, Candida spp, $T$. vaginalis y una combinación entre ellas) con relación a $C$. trachomatis. Destaca: flujo abundante, eritema vaginal o cervical y vaginosis bacteriana (VB), asociados a mediano número de casos con C. trachomatis, sin ser significativas las diferencias.

De los signos que se pudieron apreciar en genitales internos (eritema vaginal, eritema cervical, punteado rojo y ulceraciones cervicales) el que más se asoció a C. trachomatis fue el eritema (50\%).

Tabla 3. Característica del flujo vaginal, eritema, microbiota vaginal y coinfecciones en las mujeres con vaginitis según presencia de Chlamydia trachomatis

\begin{tabular}{|c|c|c|c|c|}
\hline \multirow{3}{*}{ Variables } & \multicolumn{4}{|c|}{ Chlamydia trachomatis } \\
\hline & $\mathrm{n}$ & $(-)$ & $(+)$ & p \\
\hline & & n (\%) & n (\%) & \\
\hline \multicolumn{5}{|c|}{ Cantidad de flujo } \\
\hline Escaso & 28 & $25(89,3)$ & $3(10,7)$ & \\
\hline Mediano & 68 & $58(85,3)$ & $10(14,7)$ & \\
\hline Abundante & 22 & $18(81,8)$ & $4(18,2)$ & 0,721 \\
\hline
\end{tabular}

Eritema vaginal o cervical

$\begin{array}{lllll}\text { No } & 61 & 52(85,2) & 9(14,8) & \\ \text { Sí } & 39 & 33(84,6) & 6(15,4) & \\ \text { En ambos sitios } & 19 & 16(84,2) & 3(15,8) & 1,00\end{array}$

Microbiota vaginal ${ }^{\&}$

$\begin{array}{llllll}\text { Normal } & 53 & 47(88,7) & 6(11,3) & \\ \text { Intermedia } & 22 & 20(90,9) & 2 & (9,1) & \\ \text { Vaginosis bacteriana } & 44 & 34(79,5) & 10(20,5) & 0,231\end{array}$

Coinfecciones $^{\&}$

$\begin{array}{lrrrl}\text { No } & 43 & 37(86,0) & 6(14,0) & 0,576 \\ \text { Microbiota intermedia (MI) } & 12 & 10(83,3) & 2(16,7) & 1,00 \\ \text { Vaginosis bacteriana (VB) } & 32 & 22(68,8) & 10(31,2) & 0,091 \\ \text { Candida spp. (CAN) } & 10 & 10(100,0) & 0 & 0,581 \\ \text { VB + CAN } & 9 & 9(100,0) & 0 & 0,574 \\ \text { MI + CAN } & 7 & 7(100,0) & 0 & 0,576 \\ \text { VB + T. vaginalis } & 2 & 2(100,0) & 0 & 1,00 \\ \text { MI + T. vaginalis } & 2 & 2(100,0) & 0 & 1,00 \\ \text { MI + CAN + T. vaginalis } & 1 & 1(100,0) & 0 & 1,00 \\ \text { VB + CAN + T. vaginalis } & 1 & 1(100,0) & 0 & 1,00\end{array}$

*Variables: cantidad de flujo ( 3 valores perdidos), Eritema vaginal o cervical ( 2 valores perdidos), microbiota vaginal (2 valores perdidos). \& 2 casos sin definir según Criterios de Nugent (1 caso presentó vaginits aeróbica y el otro caso no presento bacterias en el frotis). 


\section{Discusión}

El estudio realizado evaluó la presencia de $C$. trachomatis y su asociación con posibles factores de riesgo en un grupo de mujeres con vaginitis. La frecuencia global estimada de C. trachomatis fue $14,9 \%$, similar a la obtenida en mujeres embarazadas por Palma y cols. ${ }^{12}$. En el grupo etario $<36$ años se observó la mayor cantidad de casos de C. trachomatis, hecho que también ha sido reportado en estudios previos, en población adolescente $\mathrm{y}$ adulta joven ${ }^{9,17,18}$.

En este estudio, una de las variables destacables fue el número de parejas sexuales que tenían las participantes. Entre las mujeres con una pareja, con aquellas que tuvieron dos o más parejas, fue posible observar diferencia en la prevalencia de $C$. trachomatis en estas últimas ( $p=0,001)$. Como ha sido reportado por Niccolai y cols., el tener diferentes parejas sexuales o parejas sexuales no tratadas, contribuye de forma importante a presentar infecciones repetidas o reinfecciones ${ }^{19}$. Klovstad y cols., reportaron que un mayor número de parejas sexuales en los últimos 12 meses fue un factor de riesgo significativo para haber adquirido C. trachomatis ${ }^{6}$.

Otro factor analizado como relaciones sexuales oral más anal (26/119) alcanzó diferencias numéricas, aunque al límite $(\mathrm{p}=0,055)$, en contraposición con las mujeres que solamente practicaban sexo oral o anal $(p=0,311$ y $\mathrm{p}=1,00$, respectivamente). Frente a estos resultados, tener relaciones sexuales extra genitales conllevaría a una mayor posibilidad de infección por $C$. trachomatis. Como así mismo, en este grupo puede estar influyendo el alto porcentaje observado $(61,5 \%)$ de mujeres que declaró haber tenido $\geq$ dos parejas versus aquellas, que teniendo $\geq$ dos parejas, no practicaban sexo anal ni oral $(27,1 \%)$ (dato no mostrado en resultados). Yeoh y cols. observaron que tener menos de 28 años, estar casada y tener relaciones sexuales orales había aumentado significativamente las probabilidades de infección por C. trachomatis $^{20}$. En otro estudio, realizado en mujeres con relaciones anales, Llata y cols., concluyen que $20,5 \%$ de las infecciones para $C$. trachomatis se omiten cuando se examina solamente infección genital y no rectal; esto puede llevar a una transmisión continua a otras parejas o reinfección ${ }^{21}$. Si bien, en este estudio el uso de juguetes sexuales se asoció a infección por $C$. trachomatis $(\mathrm{p}=0,027)$, en la literatura médica se encuentra escasa evidencia de estudios que asocian ambos factores. Las investigaciones apuntan más a parejas homosexuales que comparten juguetes ${ }^{22}$.

En algunas de las variables analizadas (educación, uso del preservativo, microbiota vaginal), no se encontraron diferencias significativas, a pesar que la evidencia científica la ha demostrado, probablemente debido a que la muestra de este estudio fue no probabilística con un número reducido.
En relación a educación, los estudios muestran resultados contradictorios; Somayaji y cols., reportan un riesgo menor de C. trachomatis a nivel universitario con un $p=0,06^{8}$, y Huai y cols., muestran una mayor prevalencia de $C$. trachomatis en el nivel igual o mayor al universitario, con un $p=0,02^{23}$. Nuestro estudio no evidenció, $\sin$ embargo, diferencias significativas en educación, sí en parejas sexuales, como también reporta Azevedo y cols., en relación a los años de educación $(p=0,392)$ y número de parejas sexuales $(p=0,022)^{24}$. Al parecer, el nivel educacional no es un factor de riesgo consistente (como el número de parejas sexuales) y estaría dependiendo del área geográfica y de la prevalencia de C. trachomatis existente en las mujeres estudiadas.

En relación al uso del preservativo, se observó un bajo porcentaje (20,7\%) de mujeres que usaban el preservativo como una medida para prevenir las ITS. A pesar de ello, había algunas que tenían infección por $C$. trachomatis (6/25), hecho que puede estar relacionado con mal manejo del preservativo y con mayor probabilidad de contagio, razón por la cual lo utiliza. Crosby y cols., reportaron que las personas que usaron condones correctamente y consistentemente, tenían $59 \%$ menos de probabilidad de adquirir una ITS $(p=0,026)$ en comparación con aquellas que no lo hicieron ${ }^{25}$.

Cuando se analizaron las características de la microbiota, se observó que una microbiota alterada: microbiota intermedia (MI) o VB aumentaría la probabilidad de encontrar una coinfección con C. trachomatis asociada, destacando VB $(p=0,09)$. En este aspecto, Palma y cols., encontraron diferencias significativas $(p<0,05)$ en un grupo de mujeres embarazadas ${ }^{12}$.

Estos eventos (microbiota alterada o coinfección), junto a antecedentes de múltiples parejas o relaciones sexuales anales más orales, ser adolescente/adulto joven o estar embarazada, deberían ser considerados como factores de riesgo al momento de solicitar un estudio de $C$. trachomatis, sobre todo por las consecuencias conocidas que puede provocar una infección por C. trachomatis ${ }^{26}$.

Además, se ha mencionado que la biopelícula relacionada a Candida spp o G. vaginalis puede actuar como un reservorio de C. trachomatis y contribuir, tanto a la transmisión como a la diseminación de la bacteria al tracto genital superior, aumentando el riesgo de desarrollo de secuelas que afectan a la mujer en el ámbito reproductivo ${ }^{27}$. Por otro lado, se ha planteado la hipótesis que un mayor número de bacterias anaeróbicas (causantes de vaginosis bacteriana), permitiría que el interferón-gamma en estas mujeres sea incapaz de eliminar a C. trachomatis, dando como resultado infecciones repetidas o crónicas ${ }^{28}$.

En conclusión, los principales factores de riesgo asociados a C. trachomatis encontrados en este estudio, fueron el número de parejas sexuales, tener relaciones sexuales extra genitales y el uso de juguetes sexuales, 


\section{-}

mostrando sólo una tendencia la edad de inicio de actividad sexual y la coinfección con vaginosis bacteriana. Por lo tanto, siendo C. trachomatis principalmente de instalación asintomática, el conocimiento sobre factores asociados puede ayudar a identificar mujeres con alto riesgo de padecer esta infección o con altas probabilidades de llegar a contraerla. Sería altamente beneficioso incorporar en la semiología ginecológica de las mujeres consultantes, en servicios de atención primaria, los factores que se han encontrado como favorecedores de la infección por C. trachomatis ayudando con ello a la prevención, diagnóstico y manejo clínico de esta ITS.

\section{Referencias bibliográficas}

1.- Newman L, Rowley J, Hoorn S Vander, Wijesooriya N S, Unemo M, Low N, et al. Global estimates of the prevalence and incidence of four curable sexually transmitted infections in 2012 based on systematic review and global reporting. PLoS One. 2015; 10 (12) e0143304. doi: 10.1371/journal.pone.0143304.

2.- Haggerty C L, Gottlieb S L, Taylor B D, Low $\mathrm{N}, \mathrm{Xu} F$, Ness R B. Risk of sequelae after Chlamydia trachomatis genital infection in women. J Infect Dis. 2010; 201 (Suppl 2): S134-S155. doi: 10.1086/652395.

3.- Hoenderboom B M, Van Benthem B H B, Van Bergen J E A M, Dukers-Muijrers N H T M, Götz H M, Hoebe C J P A, et al. Relation between Chlamydia trachomatis infection and pelvic inflammatory disease, ectopic pregnancy and tubal factor infertility in a Dutch cohort of women previously tested for chlamydia in a chlamydia screening trial. Sex Transm Infect. 2019; 95: 300-6. doi: 10.1136/ sextrans-2018-053778.

4.- $\quad$ Price M J, Ades A E, Welton N J, Simms I, MacLeod J, Horner P J. Proportion of pelvic inflammatory disease cases caused by Chlamydia trachomatis: Consistent picture from different methods. J Infect Dis. 2016; 214 : 617-24. doi: 10.1093/infdis/jiw178.

5.- Kiguen A X, Marramá M, Ruiz S, Estofan P, Venezuela R F, Mosmann J P, et al. Prevalence, risk factors and molecular characterization of Chlamydia trachomatis in pregnant women from Córdoba, Argentina: A prospective study. PLoS One. 2019; 14 (5): e0217245. doi: 10.1371/journal.pone.0217245.

6.- Kløvstad H, Grjibovski A, Aavitsland P. Population based study of genital Chlamydia trachomatis prevalence and associated factors in Norway: A cross sectional study. BMC Infect Dis. 2012; 12: 150-7. doi: 10.1186/1471-233412-150.

7.- Bamberger D M, Graham G, Dennis L, Gerkovich M M. Extragenital gonorrhea and chlamydia among men and women according to type of sexual exposure. Sex Transm Dis. 2019; 46 (5): 329-34. doi: 10.1097/ OLQ.0000000000000967.

8.- Somayaji R, Naugler C, Guo M, Church D L. Examining sociodemographic risk factors for Chlamydia trachomatis infection: A population- based cohort study. Future Microbiol. 2017; 12 (15). doi: 10.2217/fmb-2017-0078.

9.- Silva R, León D, Viscarra T, Ili C, Roa J C, Sánchez R, et al. Frecuencia de la infección por Chlamydia trachomatis en un grupo de mujeres de la Región de la Araucanía, Chile. Rev Chil Infectol. 2013; 30 (6): 611-5. doi: 10.4067/ S0716-10182013000600006.

10.- Melo A, Lagos N, Montenegro S, Orellana J J, Vásquez A M, Moreno S, et al. Human papilloma virus and Chlamydia trachomatis by number of sexual partners and time of sexual activity on university students in the Region of La Araucanía, Chile. Rev Chil Infectol. 2016; 33 (3): 287-92. doi: 10.4067/S071610182016000300006.

11.- Ovalle A, Martínez M A, de la Fuente F, Falcon N, Feliú F, Fuentealba F, et al. Prevalencia de infecciones de transmisión sexual en mujeres embarazadas atendidas en un hospital público de Chile. Rev Chilena Infectol. 2012; 29 (5): 517-20. doi: 10.4067/S071610182012000600006.

12.- Palma C, Martínez M A, Santander E. Screening of cervical sexually transmitted infections in pregnant women and the relation with the vaginal microbiota. Rev Chilena Infectol. 2019; 36 (3): 292-8. doi: 10.4067/ S0716-10182019000300292.

13.- Rowley J, Hoorn S Vander, Korenromp E, Low N, Unemo M, Abu-Raddad L J, et al. Chlamydia, gonorrhoea, trichomoniasis and syphilis: Global prevalence and incidence estimates, 2016. Bull World Health Organ. 2019; 97 (8): 548-62. doi: 10.2471/ BLT.18.228486

14.- Nugent R P, Krohn M A, Hillier S L. Reliability of diagnosing bacterial vaginosis is improved by a standardized method of Gram stain interpretation. J Clin Microbiol. 1991; 29 (2): 297-301. PMID: 1706728.

15.- Jordá G B, Hanke S E, Ramos-Rincón J M, Mosmann J, Lopéz M L, Entrocassi AC, et al. Prevalence and phylogenetic analysis of Chlamydia trachomatis in a population of women in Posadas, Misiones. Rev Esp Quimioter. 2018; 31 (1): 21-6. PMID: 29451375. Disponible en: https://europepmc. org/backend/ptpmcrender.fcgi?accid=PMC6159 361\&blobtype $=$ pdf.

16.- Kengne P, Veas F, Vidal N, Rey J L, Cuny G. Trichomonas vaginalis: repeated DNA target for highly sensitive and specific polymerase chain reaction diagnosis. Cell Mol Biol (Noisy-le-grand). 1994; 40 (6): 819-31. PMID: 7812190.

17.- Miranda A E, Silveira M F, Travassos A G, Tenório T, Val I C C do, Lannoy L de, et al. Prevalence of Chlamydia trachomatis and Neisseria gonorrheae and associated factors among women living with human immunodeficiency virus in Brazil: a multicenter study. Braz J Infect Dis. 2017; 21 (4): 402-7. doi: 10.1016/j.bjid.2017.03.014.

18.- De Borborema-Alfaia A P B, De Lima Freitas N S, Filho S A, Borborema-Santos C M. Chlamydia trachomatis infection in a sample of northern Brazilian pregnant women: Prevalence and prenatal importance. Braz J Infect Dis. 2013; 17 (5): 545-50. doi: 10.1016/j. bjid.2013.01.014.

19.- Niccolai L M, Livingston K A, Laufer A S, Pettigrew M M. Behavioural sources of repeat Chlamydia trachomatis infections: Importance of different sex partners. Sex Transm Infect. 2011; 87 (3): 248-53. doi: 10.1136/ sti.2010.045484.

20.- Yeoh C A, Chan L C, Ch'ng C C, Tan W C. Prevalence and risk factors of genitourinary Chlamydia trachomatis infection among patients attending sexually transmitted disease clinics in northern Malaysia. Med J Malaysia. 2020; 75 (2): 103-9. Disponible en: https:// europepmc.org/article/med/32281589

21.- Llata E, Braxton J, Asbel L, Chow J, Jenkins L, Murphy R, et al. Rectal Chlamydia trachomatis and Neisseria gonorrhoeae infections among women reporting anal intercourse. Obstet Gynecol. 2018; 132 (3): 692-7. Consultado 12 mayo 2021. Disponible en: https://pubmed. ncbi.nlm.nih.gov/30095784/

22.- Marrazzo J M, Gorgos L M. Emerging sexual health issues among women who have sex with women. Curr Infect Dis Rep. 2012; 14 (2): 20411. doi:10.1007/s11908-012-0244-x.

23.- Huai P, Li F, Li Z, Sun L, Fu X, Pan Q, et al. Prevalence, risk factors, and medical costs of Chlamydia trachomatis infections in Shandong Province, China: A population-based, crosssectional study. BMC Infect Dis. 2018; 18 (534): 1-11. doi: 10.1186/s12879-018-3432-y.

24.- De Azevedo M J N, Dos Santos Nunes S, De Oliveira F G, Pires Rocha D A. High prevalence of Chlamydia trachomatis in 
pregnant women attended at primary health care services in amazon, Brazil. Rev Inst Med Trop Sao Paulo. 2019; 61:e6. doi: 10.1590/ S1678-9946201961006.

25.- Crosby R A, Charnigo R A, Weathers C, Caliendo A M, Shrier L A. Condom effectiveness against non-viral sexually transmitted infections: A prospective study using electronic daily diaries. Sex Transm
Infect. 2012; 88 (7): 484-9. doi: 10.1136/ sextrans-2012-050618.

26.- Price M J, Ades A E, Soldan K, Welton N J, Macleod J, Simms I, et al. The natural history of Chlamydia trachomatis infection in women: A multi-parameter evidence synthesis. Health Technol Assess. 2016; 20 (22): 1-250. doi: 10.3310/hta20220.

27.- Filardo S, Di Pietro M, Tranquilli G, Sessa R.
Biofilm in genital ecosystem: a potential risk factor for Chlamydia trachomatis infection. Can J Infect Dis Med Microbiol. 2019; Article ID 1672109. doi:10.1155/2019/1672109.

28.- Ziklo N, Huston W M, Hocking J S, Timms P. Chlamydia trachomatis genital tract infections: when host immune response and the microbiome collide. Trends Microbiol. 2016; 24 (9): 750-65. doi:10.1016/j.tim.2016.05.007. 